\title{
Bioética y buen gobierno en la Union Europea
}

\section{Bioethics and good governance in the European Union}

\author{
MARIA TERESA LÓPEZ DE LA VIEJA \\ Universidad de Salamanca
}

Recibido: 05-12-2008 Aprobado definitivamente: 16-01-2009

\section{RESUMEN}

La Carta de Derechos Fundamentales de la Unión Europea contribuye a desarrollar la ciudadanía europea, basada en valores comunes, como la justicia y la dignidad. La Carta se refiere al consentimiento libre e informado en Medicina y Biología, prohibiendo la clonación reproductiva y las prácticas eugenesicas. El artículo considera el papel de los temas de Bioética en la expansión de los derechos de los ciudadanos; los debates recientes han demostrado que los derechos han de estar garantizados y, además, que las buenas practicas profesionales sólo pueden desarrollarse dentro de buenas instituciones. El artículo analiza el papel de los principios de gobernanza (transparencia, eficiencia, participación, rendición de cuentas), así como la relación interna entre éstos y la integración de las políticas publicas: las cuestiones de Bioética ejemplifican el proceso de «europeanización».

\section{PALABRAS CLAVE \\ BIOÉTICA, UNIÓN EUROPEA, GOBERNANZA, BUENAS PRACTICAS, EUROPEANIZACIÓN}

\begin{abstract}
The Charter of Fundamental Rights of the European Union contributes to develop European citizenship based on common values like justice and dignity. The Charter refers to free and informed consent in Medicine and Biology, and prohibits reproductive cloning and eugenic practices. This article considers the role of bioethical topics in the expansion of citizens' rights; in fact, recent debates not only have proved that rights have to be guaranteed but that good professional practice could only develop within good institutions. The article also analyzes the role of the principles of governance (transparency, efficiency, participation, accountability), and the internal relationship between these principles and the integration of social policies; bioethical issues could exemplify the process of Europeanization.
\end{abstract}

C Contrastes. Revista Internacional de Filosofía, vol. XIV (2009), pp. 179-198. ISSN: 1136-4076 Licenciatura de Filosofía, Universidad de Málaga, Facultad de Filosofía y Letras Campus de Teatinos, E-29071 Málaga (España) 


\author{
KEY WORDS \\ BIOETHICS, EUROPEAN UNION, GOVERNANCE, GOOD PRACTICES, \\ EUROPEANIZATION
}

\title{
DERECHO A LA INTEGRIDAD DE LA PERSONA
}

1. Toda persona tiene derecho a su integridad física y psíquica.

2. En el marco de la medicina y la biología se respetarán en particular:

(a) el consentimiento libre e informado de la persona de que se trate, de acuerdo con las modalidades establecidas en la ley,

(b) la prohibición de las prácticas eugenésicas, y en particular las que tienen por finalidad la selección de las personas,

(c) la prohibición de que el cuerpo humano o partes del mismo en cuanto tales se conviertan en objeto de lucro,

(d) la prohibición de la clonación reproductora de seres humanos.

Carta de Derechos Fundamentales de la Unión Europea 2007/C303/01, art. 3 .

El DERECHO a LA INTEGRIDAD ESTÁ RECOGIDO en el Carta de Derechos para los ciudadanos de la Unión Europea. El documento se refiere en modo expreso al consentimiento informado, a las prácticas eugenésicas y a la clonación, temas especializados que son, además, motivo de desacuerdos y de intensos debates tanto en el campo de la Medicina y la Biología como en la opinión pública. Por ello, resulta significativo que un documento de tal rango haya incluido estas cuestiones entre los derechos básicos y comunes para todos los ciudadanos de la Unión. Hay que tener en cuenta asimismo el hecho de que el consentimiento y la prohibición de determinadas prácticas han sido ya regulados por la mayoría de los países de la Unión, por lo que convendría explicar por qué razones el respeto por la integridad individual y el rechazo hacia ciertas técnicas ocupan un lugar tan destacado en un texto que presenta a la Unión Europa como un espacio de libertad, seguridad y justicia. En las páginas siguientes se considera que la actual regulación de los temas de Bioética desde las instituciones europeas es un ejemplo significativo. En primer lugar, responde a la tendencia a extender los derechos de los ciudadanos, a fin de proteger sus libertades y garantizar su bienestar. Las garantías al estilo europeo tienen mucho que ver con las nuevas responsabilidades de la ciencia y, sin duda, con experiencias negativas del pasado, que no deberían repetirse. En segundo término, esto sucede en un momento clave para la construcción de un modelo político, modelo que descansa o pretender 
descansar sobre los principios básicos de un buen gobierno o gobernanza. Por último, el estado de las políticas que conciernen a la salud, la práctica clínica y la investigación biomédica ejemplifican una forma característica de integración, a varios niveles y conforme al método comunitario.

En los años precedentes, esta forma de cambio institucional ha sido denominada «europeanización», uno de cuyos rasgos consiste en mantener estructuras distintas dentro del mismo sistema. En general, la integración al estilo europeo responde al propósito de construir un espacio de libertad, seguridad y justicia para todos los individuos, ${ }^{1}$ tal como se declara en la Carta de Derechos Fundamentales. A la vez, esta forma característica de abordar los temas de salud y el uso de las técnicas biomédicas demuestra que la integración de los países y de las políticas puede realizarse a distintos niveles. Se realiza, de hecho, a varios niveles con resultados dispares, aún habiendo seguido una misma dirección. Cabe decir entonces que, más allá del significado político de la ampliación, de los tratados y de las instituciones europeas, todo lo relativo a la salud y a la investigación médica y biológica resulta apropiado para evaluar el alcance y las posibilidades que ofrece un modelo de ciudadanía y de integración. Se trata de un modelo normativo, de «buen gobierno», que, en la práctica, están siguiendo las instituciones europeas para regular determinados sectores o políticas publicas. De acuerdo con esto, la protección de la salud y de la integridad, tal y como se está realizando en la mayoría de los países de la Unión, indica, entre otras cosas, que lo personal es también un asunto político de primer orden.

\section{DeRechos Cívicos}

Dentro de la Unión, es preciso aproximar las Instituciones europeas al ciudadano. Sin duda alguna, los ciudadanos siguen respaldando los grandes objetivos de la Unión, pero no siempre perciben la relación entre dichos objetivos y la actuación cotidiana de la Unión. Desean unas Instituciones europeas menos lentas y rígidas y, sobre todo, más eficientes y transparentes. Muchos piensan también que la Unión debería prestar mayor atención a sus preocupaciones concretas. $^{2}$

1 «Los pueblos de Europa, al crear entre sí una unión cada vez más estrecha, han decidido compartir un porvenir pacífico basado en valores comunes.

Consciente de su patrimonio espiritual y moral, la Unión está fundada sobre los valores indivisibles y universales de la dignidad humana, la libertad, la igualdad y la solidaridad, y se basa en los principios de la democracia y del Estado de Derecho. Al instituir la ciudadanía de la Unión y crear un espacio de libertad, seguridad y justicia, sitúa a la persona en el centro de su actuación» Carta de Derechos Fundamentales de la Unión Europea 2007/C303/01, Preámbulo.

2 Declaración de Laeken, 15-12-2001. 
1) Una de las principales dificultades para el avance efectivo de la convergencia política en la Unión está en la percepción que, a menudo, los ciudadanos tienen de las instituciones y, en general, de la forma en que se toman las decisiones «en Bruselas». En el 2001, la Declaración de Laeken señalaba que esta visión negativa es uno de los principales obstáculos para la participación efectiva de todos en el proyecto europeo, ya que las instituciones parecen funcionar al margen de los intereses y de la vida cotidiana de los mismos ciudadanos. Existe un importante déficit democrático, ésta era la conclusión. Era y sigue siendo de un grave inconveniente para la consolidación de la Unión como proyecto de carácter democrático y, sin duda, este déficit erosiona tanto las instituciones como la legitimidad de las decisiones que se toman en la Comisión Europea o en el Parlamento. Por un lado, los ciudadanos esperan que el nuevo marco institucional contribuya a una mejor solución de los problemas que les afectan día a día, por otro mantienen una actitud distante, cuando no una actitud de desconfianza hacia esas mismas instituciones supranacionales. El problema inquieta a los representantes políticos y a los responsables de la Unión, como demostraba el Libro Blanco ${ }^{3}$ sobre la política europea, dado a conocer ese mismo año, en el 2001. Por éstos y varios motivos más, habría que tomar en serio la cuestión planteada por la Declaración de Laeken, a fin de aproximar las instituciones a los ciudadanos, en beneficio de éstos y del buen gobierno de la Unión.

2) La salud y los recursos para protegerla y promoverla son temas que forman parte de la existencia cotidiana y, al mismo tiempo, están en la agenda de los Estados nacionales, que son los responsables directos de los sistemas de salud y de los servicios públicos. Los sistemas son muy diferentes en los países europeos, a día de hoy no existe la integración en esta área, siendo deseable que se produzca a corto o medio plazo. Con todo, lo relativo a la salud permite entender mejor cómo funciona la convergencia y cómo interactúan en la práctica los tres niveles de lo cívico que están en el proyecto europeo: la expansión de derechos, la demanda creciente de buenas prácticas, la integración por segmentos, asimétrica, dentro de la Unión. A diferencia de lo que viene ocurriendo con otras áreas, la protección de la salud ha vertebrado algunos de estos procesos, también las formas de negociación y, sin duda, ciertos avances en la legislación de los países europeos sobre la materia. En general, ha favorecido la convergencia de las instituciones, generando un tipo de acuerdos que van en la dirección correcta, entre lo supranacional y la cercanía a los ciudadanos, a sus necesidades básicas. Tal vez, los esfuerzos por crear un espacio común de seguridad y de libertades resulten más visibles para la opinión pública, a pesar de lo cual la adaptación mutua en materia de salud y de investigación biomédica demuestra

3 Comisión de las Comunidades Europeas, La gobernanza europea. Un libro blanco, COM (2001) 428 final. 
que es posible aplicar los criterios generales de buen gobierno respetando, al mismo tiempo, la diversidad y la subsidiaridad en la toma de decisiones.

3) Además de esto, los avances en las políticas de salud constituyen un precedente muy útil a la hora de afrontar de forma coordinada algunos temas clave - desde la seguridad alimentaria, la donación de órganos y tejidos, hasta la investigación con sujetos humanos y con los no humanos-, antes de contar con un marco institucional bien definido. Por así decirlo, la Europa de la salud demuestra que las políticas de alcance europeo son posibles en este momento, aunque todavía no exista ni la Constitución ni la Europa supranacional, proyectos que requieren negociaciones bastante complejas. En cambio, lo relativo a la salud influye de forma inmediata en la calidad de vida individual y en el desarrollo de las sociedades; en este caso, ejemplifica además un tipo de convergencia que es discontinua, fragmentaria y, a pesar de todo, demuestra que la Unión es y seguirá siendo un espacio sin fronteras. Esto es, la investigación biomédica se ha convertido en uno de esos asuntos que más dividen a los europeos y, a la vez, en una de las áreas más reguladas en Europa. Servirá ahora para examinar varios aspectos que influyen tanto en la vida de los ciudadanos como en la convergencia de las instituciones. Tiene que ver con la protección de los derechos fundamentales, el creciente interés por establecer un marco de buenas prácticas a fin de distanciarse para siempre de episodios negativos de la Historia, un marco para hacer frente también a los problemas que afectarían a futuras generaciones y, por último, tiene que ver con el interés por definir la convergencia al estilo europeo.

\section{I.1. LA INVESTIGACIÓN BIOMÉDICA}

[...] [S]e autoriza la utilización de cualquier técnica de obtención de células troncales humanas con fines terapéuticos o de investigación, incluida la activación de ovocitos mediante transferencia nuclear, que no comporten la creación de un preembrion o de un embrión. ${ }^{4}$

La Ley 14/2007 regula algunos temas que han resultado controvertidos a lo largo de los últimos años, como el uso de las técnicas genéticas, el acceso a datos genéticos de carácter personal y la utilización de células troncales para la investigación. La Ley sólo admite este tipo de ensayos cuando tienen una finalidad terapéutica, fijando las condiciones y las garantías para acceder a las técnicas para la obtención de células humanas. Conviene recordar que ya existían leyes aprobadas con anterioridad, como la Ley 14/2006 sobre técnicas de reproducción asistida - para regular la inseminación artificial, la fecundación in

4 LEY 14/2007 de Investigación biomédica, Preámbulo, IV. 
vitro, la transferencia de gametos-, cuyo objetivo era asegurar tanto la autonomía como la seguridad de los individuos que acceden a los tratamientos. La regulación de tales técnicas indica que existe en la sociedad española suficiente nivel de consenso en estos temas, a pesar de que sigan siendo objeto de desacuerdo y motivo de polémicas ideológicas. El propósito terapéutico justifica el uso de este tipo de células para la investigación, siempre dentro de un marco que asegure el respeto por las libertades y por los derechos fundamentales. Conviene recordar, en segundo término, que la legislación nacional en la materia ha ido más allá que la de otros países europeos, manteniendo un enfoque similar al ya adoptado en el Reino Unido, estando en cambio bastante alejada de las posiciones defendidas en Alemania a este respecto hasta hace poco.

Con todo, la ley sobre investigación biomédica sigue algunos de los principios defendidos en los acuerdos, informes, directivas y recomendaciones vigentes en la Unión, que algunos países no han trasladado todavía a su legislación, por varios motivos, tanto técnicos como ideológicos. En este caso, han funcionado el respeto por las diferencias y la «geometría variable» del proyecto europeo. Por esta misma razón, la Ley 14/ 2007 está dentro del marco normativo general que han ido construyendo los representantes europeos para las cuestiones de Bioética; se trata de un marco de principios básicos, también de pluralismo ideológico, compatible con la existencia de reglas de actuación para los profesionales que han de tomar decisiones en situaciones complicadas.

(a) Los principios generales a seguir por los investigadores se encuentran en el Convenio ${ }^{5}$ de 1997 sobre derechos humanos y Biomedicina, así como en la Carta de Derechos Fundamentales de la Unión Europea, en su primera versión del año 2000 y en el texto definitivo, dado a conocer en el 2007. A su vez, este marco normativo sigue de cerca los criterios defendidos con anterioridad, desde 1949 en el Código de Nürnberg y, luego, en la Declaración de Helsinki en los años sesenta y en versiones de años posteriores. Por lo tanto, existe coherencia entre los documentos que se refieren a la práctica clínica y a la investigación científica, ya que el objetivo fundamental ha sido y sigue siendo la protección de los derechos individuales en esta área. De hecho, desde 1949 se ha intentado consolidar un modelo de ciencia responsable, al servicio de los intereses y las necesidades de los individuos, por encima de los intereses de la sociedad o los de la misma ciencia. Con este objetivo, el respeto por la integridad y la dignidad de los pacientes o de los sujetos de la experimentación ha llegado a ser principios aceptados por todos en el momento de regular todo lo relativo al ámbito biomédico. Este hecho no impide que las normas nacionales desarrollen sólo alguno de los aspectos o sigan reglas diferentes en asuntos especialmente controvertidos.

5 Convenio para la protección de los derechos humanos y la dignidad del ser humano con respecto a las aplicaciones de la Biología y la Medicina, Oviedo, 1997. 
(b) Valga como ejemplo del pluralismo europeo en temas de Bioética la forma de abordar y de regular la clonación con fines terapéuticos. No hay duda de que el asunto ha merecido una especial atención en los países de la Unión, como demostraba ya la Resolución del Parlamento del año 1988. Varios documentos posteriores hacen referencia a la misma cuestión, respondiendo a la diversidad de puntos de vista y de creencias entre los europeos; según esto, se aceptan algunos principios comunes pero éstos se aplican de manera diferente en cada país. Esta circunstancia explica el hecho de que la Ley 14/2007 sea bastante similar a la legislación del Reino Unido y, en cambio, tenga sólo algunos elementos en común con la normativa vigente en otros países de la Unión. Existen más ejemplos de lo mismo, la existencia de acuerdos sobre el criterio general y, luego, desacuerdos en temas especiales. A pesar de esto, hay un «estilo europeo»-si así puede llamarse-, que consiste en regular, en definir los requisitos, los procedimientos, las competencias y los controles que resultan necesarios en el campo de la Medicina y de la Biología. El ideal de ciencia responsable está expresamente ligado a la calidad ${ }^{6}$ y a la adopción de «buenas prácticas», ${ }^{7}$ demostrando así que, más allá del respeto por los derechos individuales, hace falta el compromiso de los investigadores y de las instituciones con los derechos y las libertades. En suma, el modelo se halla entre pluralismo y la afirmación de estándares comunes, bien definidos, para asegurar la calidad de los procedimientos y de los resultados. Se trata, sin duda, de estándares científicos y morales, puesto que la eficacia en la investigación ha de ser compatible con la seguridad y con la igualdad de oportunidades, permitiendo, por ejemplo, que todos tengan acceso a los beneficios de los ensayos o de los tratamientos resultantes de la investigación, en igualdad de condiciones.

(c) El consentimiento informado garantiza que la actuación de los profesionales de la salud y de los investigadores respetará la autonomía de los sujetos. Se entiende, entonces, por qué los convenios y directrices sobre ensayos y sobre investigación con seres humanos, en Europa pero también fuera de Europa -como sucede con las directrices del OMS del año 2002 y el Protocolo Adicional al Convenio de Oviedo, del año 2005-, cuentan con este procedimiento y tienden a ampliarlo: el consentimiento informado representa una garantía para los ciudadanos. Así es en el caso de quienes no están en condiciones ni tienen la capacidad para valorar con plena independencia los riesgos de los tratamientos y de los ensayos, como ocurre a veces con los menores de edad o con las personas privadas de libertad. Es más en tales casos, la regulación del consentimiento ha

6 LEY 14/2007 de Investigación biomédica, Promoción y calidad de la investigación biomédica, art. 10

7 López de la Vieja, M. T.: «Ética de la investigación. Las buenas practicas», Arbor, $730,2008,233-245$ 
de ser mucho más precisa que en el ámbito clínico, debido a la vulnerabilidad de los individuos carentes de autonomía efectiva, sin posibilidad de ponderar adecuadamente los riesgos de los ensayos.

Hay un motivo adicional para insistir en el respeto por los derechos y en el rigor de los procedimientos: los malos precedentes, los precedentes trágicos. ${ }^{8}$ En Europa, el enfoque liberal -la prioridad de los derechos individuales- se ha traducido en reglamentación, leyes, acuerdos explícitos, convenios, creación de comités, etc., a causa de lo ocurrido en una etapa reciente de la Historia. El pasado ha demostrado que las malas prácticas y la vulneración de los derechos fundamentales llevan a abusos y a conductas infames, dejando sin protección a los sujetos más vulnerables. Los precedentes, los malos precedentes de la Medicina y la Biología sometidas al control de un sistema totalitario, explican también las fuertes reacciones que aún suscitan en Alemania la aplicación de las nuevas técnicas biomédicas o la posibilidad de una nueva eugenesia. Los países del mismo entorno no cuentan con un pasado tan abrumador, pero asumen que es necesario garantizar la completa protección de los derechos de quienes participan en la experimentación biomédica, tanto los sujetos competentes como, ante todo, los que requieren del consentimiento de sus representantes legales. Los precedentes y, en general, la eventualidad de que se produzcan malas prácticas, trato abusivo, excesos, justifican que la Carta de Derechos Fundamentales del año 2007 se refiera, una vez más, al consentimiento informado, así como al rechazo de la clonación y de las prácticas eugenésicas (art.3).

\section{EL «BUEN GOBIERNO»}

La Ley 14/2007 presta también atención a los comités de Ética, por entender que éstos favorecen la intervención de la sociedad civil en la toma de decisiones. La deliberación en los comités responde al enfoque multidisciplinar y plural que ha de seguir la investigación biomédica. Además de esto, contribuye a que las prácticas sean transparentes y, por ello, significa un paso más hacia el «buen gobierno» o gobernanza en la práctica clínica y en la investigación científica. La ciencia es una institución que, como otras muchas instituciones, ha de estar abierta al escrutinio de los ciudadanos, afectados tanto por los beneficios como por los riesgos de la experimentación. En consecuencia, la función de la Ética en el ámbito de la investigación consiste, sobre todo, en definir los principios que ayuden a discriminar entre las malas y las buenas prácticas, trazando asî fronteras entre lo que se debe y lo que no se debe hacer en este ámbito. No cabe

8 López de la Vieja, M. T.: «Posguerra y contracultura. Identidades en la Ética aplicada», Arbor, 722, 2006, 787-795 
duda de que la eficacia es un criterio fundamental para evaluar los resultados de la ciencia, sólo que estos resultados han de ser compatibles con la responsabilidad, la justicia y, en suma, con el buen gobierno o gobernanza en todas las fases de la investigación.

¿Qué se entiende por gobernanza? Según el documento elaborado en el año 2001 por la Comisión de las Comunidades Europeas, el término se refiere básicamente a «cómo las cosas podrían y deberían hacerse». ${ }^{9}$ Este concepto, de carácter normativo, vale tanto para los procedimientos, las prácticas como para las normas y, en suma, para todas las formas de ejercer el poder dentro de la Unión. El interés por el tema estaba motivado en parte por la dificultad ya señalada, la necesidad de aproximar las instituciones comunitarias a las demandas de los ciudadanos, con la intención de reforzar la legitimidad y, en último término, la estructura democrática de la representación y el gobierno de los europeos. La Declaración de Laeken era muy clara a este respecto; sin duda alguna, el futuro del proyecto político común dependerá del respaldo que le otorguen los mismos ciudadanos, de su confianza en las instituciones comunes. Por ello, las reformas deberían ir en esa línea: las actuaciones, la legislación, los organismos, los responsables políticos tendrán siempre en cuenta «cómo las cosas podrían y deberían hacerse».

Dentro de este enfoque normativo, los principales requisitos de buen gobierno o gobernanza son cinco: apertura, participación, responsabilidad, eficacia y coherencia. Mayor apertura significa que es necesario promover una comunicación más activa entre instituciones y ciudadanos, no sólo para favorecer el uso de un lenguaje accesible a todos sino, ante todo, para lograr la confianza de los ciudadanos. Su participación será la condición fundamental para que el diseño y la aplicación de las políticas comunes tengan el respaldo de los europeos. En cuanto a la responsabilidad -la de todos, los Estados y los agentes- obliga a clarificar los procesos, a todos los niveles. No hay duda de que los ciudadanos esperan que se actúe con eficacia, de modo que las intervenciones produzcan resultados, partiendo de objetivos claros y con una adecuada evaluación del impacto que puedan tener las decisiones. Por último, la coherencia resulta especialmente necesaria en actuaciones y procedimientos que resultan en extremo complejos en el marco de la Unión; así lo demuestran problemas de tanto alcance como son el cambio demográfico y el cambio climático.

En la Unión Europea existen varios niveles y distintas formas de intervención política, como no podía ser de otra manera, dadas las características y la Historia de los europeos. Al mismo tiempo, la plena integración de instituciones y de agentes resulta decisiva para consolidar el proyecto político, de modo que el

9 Comisión de las Comunidades Europeas, La gobernanza europea. Un libro blanco, COM (2001) 428 final. 
proceso de convergencia tendría que ir en una dirección bien definida y que fuese aceptada por todos. La gobernanza o buen gobierno indica esto mismo, una forma de organizar las actuaciones políticas de acuerdo con el método comunitario; es decir, combinando la apertura con la coherencia interna. ${ }^{10}$ ¿Cómo se lleva a la práctica este tipo de actuaciones? Por un lado las instituciones ${ }^{11}$ evolucionan para crear una política trasnacional $\mathrm{o}$, al menos, una política sin fronteras dentro de la Unión. A su vez, esto genera interdependencia, tipos de coordinación institucional que coexisten con la autonomía de los estados nacionales. En tal sentido, la gobernanza produce redes e intervenciones abiertas, descentradas pero estructuradas. El «intergubernamentalismo» ${ }^{12}$ sería uno de los rasgos de las políticas genuinamente europeas, encaminándose, mal que bien, hacia una soberanía compartida ${ }^{13}$ como objetivo básico. Por otro lado, existen aún y seguirán existiendo formas dispares de organización, de agencias, de procedimientos, que permiten establecer intercambios y redes no jerárquicas, flexibles.

Con esta dinámica compleja, la fragmentación resulta inevitable, sobre todo por el papel central que aún conservan los estados nacionales en la Unión. Todo ello complica el proceso e incluso la manera de entender el proyecto político trasnacional, ¿cómo se define, qué es y que va a ser la Unión Europea? La «geometría variable» es una de sus principales características, aunque, según la expresión empleada en alguna ocasión por J. Delors, se trata más bien de un «objeto político no identificado». ${ }^{14}$ ¿Tendrá la forma de una organización intergubernamental? ¿Habrá un Estado europeo único, supranacional? ¿Será una federación de estados nacionales? En las actuales circunstancias, no resulta probable la existencia de algo así como los Estados Unidos de Europa. Por el momento, la Unión se asemeja ${ }^{15}$ más bien a lo que percibirían varias personas con problemas de visión, con ceguera: al tocar un elefante, cada una describiría un animal distinto.

10 Comisión de las Comunidades Europeas, La gobernanza europea. Un libro blanco, COM (2001), 428 final.

11 Wallace, H., Wallace, W., Pollack, M.: «An Overview», Policy-Making in the European Union, Oxford University Press, Oxford, 2005, 3-11.

12 Pollack, M.: «Theorizing EU Policy-Making», Wallace, H., Wallace, W., Pollack, M.: Policy-Making in the European Union, 13-48.

13 W.Wallace ha preferido usar el término «post soberanía» para describer este fenomeno, «Post-soverign Governance: The EU as a Partial Polity», Wallace, H., Wallace, W., Pollack, M.: Policy-Making in the European Union, 483-503.

$14 \mathrm{Ph}$. Schmitter ha tenido en cuenta ésta y otras paradojas del proyecto politico europeo, «Examining the Present Euro-Polity with the Help of Past Theories», Marks, G., Scharpf, F. Schmitter, Ph.: Governance in the European Union, Sage, London, 1996, 1-14.

15 M. Pollack recordaba la imagen que había sido ya empleada por D. Puchala en 1972, «Theorizing EU Policy-Making», Wallace, H., Wallace, W., Pollack, M.: Policy-Making in the European Union, 45. 


\section{II.1. GOBERNANZA TRASNACIONAL}

El elemento normativo sirve para articular todos los niveles, lo vertical y lo horizontal, lo formal y lo informal en la actual política al estilo europeo. La gobernanza permite, en efecto, que intervengan múltiples agentes, que la deliberación esté abierta y transcurra en niveles y foros bien distintos, que las negociaciones sean multilaterales, que las prácticas comunes y las instituciones sean siempre flexibles y, en definitiva, que las fronteras, todo tipo de fronteras, vayan desapareciendo en el espacio de la Unión. Ahora bien, los procedimientos y las prácticas - por flexibles y abiertos que lleguen a ser- se han de guiar por los principios generales del buen gobierno, con objeto de construir instituciones que merezcan la confianza de los ciudadanos y animen a la participación en los asuntos comunitarios. Los tratados de la Unión, sobre todo después del de Maastricht, en 1991, han intentado clarificar las reglas, las competencias, los procedimientos de un proceso necesariamente complejo y, en ocasiones, con ciertas dosis de improvisación. ${ }^{16}$ Por esta razón, hacen falta algunos criterios generales, como la rendición de cuentas, un horizonte trasnacional y, ante todo, un método lo suficientemente flexible:

- Algo básico para llegar a una forma de autogobierno que propicie la intervención de la sociedad civil y que, a la vez, consolide instituciones transparentes, dignas de crédito, será la capacidad para rendir cuentas (accountability) $)^{17}$ en el espacio público. El buen gobierno estará, pues, abierto al escrutinio y a la intervención de todos los interesados, dar cuenta resulta, pues, obligado para agentes e instituciones que aspiran a la máxima transparencia y a la legitimidad. Es cierto que el crecimiento de la influencia de los ciudadanos en los sistemas democráticos, así como el pluralismo en las formas de ejercer el liderazgo y varios cambios en la misma dirección -y otros cambios que están aun por llegar- suponen un avance decidido hacia lo se conoce ya como «gobernanza sin gobierno». ${ }^{18}$ Sin embargo, esta forma moder-

$16 \mathrm{Ph}$. Schmitter ha comentado ambos aspectos, tanto los avances realizados en la «europolítica» como las actuaciones improvisadas a lo largo del proceso de integración, «Examining the Present Euro-Polity with the Help of Past Theories», Marks, G., Scharpf, F., Schmitter, Ph.: Governance in the European Union, 1-14.

17 M. Teló vincula los mecanismos de control por parte de los ciudadanos a la defensa de algo tan fundamental como los derechos humanos, «Civilian Power and Interntional Relations: the EU and the Multilateralism from the Twentieth to the Twenty-First Century», Europe: A Civilian Power?, Palgrave Mac Millan, London, 2006, 1-105.

18 Teló, M.: «The Process of Treaty Reform: The International Dimension», Europe: A Civilian Power?, 252-277. 
na y abierta de organizar las relaciones en la esfera pública requiere también rigor en los mecanismos de control. Un control a realizar tanto a escala regional y nacional como en el nivel internacional. $\mathrm{La}$ principal razón para extremar los controles -por ejemplo, en la lucha contra la corrupción- es que el buen gobierno está relacionado con el desarrollo y con la lucha contra la pobreza, tal como afirmaba en el año 2001 el entonces Presidente de la Comisión Europea, R. Prodi. ${ }^{19}$ En conclusión, las dimensiones múltiples de la actividad política, la carencia de autoridad central e incluso el ritmo desigual, marcado por la «geometría variable» ${ }^{20}$ de la construcción europea, estas peculiaridades no deberían impedir la toma de decisiones que sean correctas, estén plenamente justificadas y sean siempre transparentes.

- Los principios de buen gobierno son más relevantes si cabe en la Unión, un proyecto de integración que, por necesidad, se tiene que distanciar del modelo nacionalista a fin de tener resultados positivos a medio y a largo plazo. En ausencia de un pueblo, un demos europeo, la convergencia de países, instituciones, agentes y formas de proceder tan variadas sólo puede guiarse por un modelo cosmopolita, abierto, alejado, por tanto, de las versiones nacionalistas del intergubernamentalismo europeo. El desarrollo de instituciones comunes y de formas de cooperación es aún muy desigual, bastante fragmentario, sin embargo muestra que la negociación y el poder ejercido de forma democrática y participativa son también efectivos. Es más, ese estilo de gobernar «sin gobierno» representa una oportunidad para poner en marcha un modelo cosmopolita, ${ }^{21}$ desde el respeto por las diferencias y algunos principios de validez universal. La actual expansión de los procesos globales es un argumento más - un argumento de peso- para hacer valer este enfoque normativo de lo político - con justicia e igualdad ${ }^{22}$ - en las instituciones que, cada vez más, tienen una dimensión supranacional.

19 Alocución de Romano Prodi, Presidente de la Comisión Europea, Inauguración de la « ${ }^{\text {a }}$ Conferencia de las Naciones Unidas sobre los Países Menos Adelantados», Bruselas, 14 de mayo de 2001.

$20 \mathrm{Ph}$. Schmitter ha señalado los distintos niveles de agregación, local, subnacional, nacional, «Imagining the Future of the Euro-Polity with the Help of New Concepts», Marks, G., Scharpf, F., Schmitter, Ph.: Governance in the European Union, 121-165.

21 Sobre el proyecto social y político europeo, abierto y de carácter cosmopolita, Beck, U., Grande, E.: «Einleitung: Die europäische Malaise und warum die Idee des kosmopolitische Europa sie überwinden könnte», Das kosmopolitische Europa, Suhrkamp, Frankfurt, 2004, 9-48.

22 Las bases éticas de la globalización han sido analizadas por D. Held y A. McGrew, «World Orders, Ethical Foundations», Globalization/Anti-Globalization, Polity Press, Cambridge, 2002, 77-87. 
Esto es, la Unión puede ser un banco de pruebas para los cambios sociales y políticos que resultan necesarios en un mundo sin fronteras.

- Tal vez el auténtico valor añadido del proyecto europeo reside en su capacidad para difundir buenas prácticas en todos lo sectores y países de la Unión, sin anular las competencias propias de los estados europeos. La «geometría variable» demuestra así su lado más favorable, ya que puede combinar las políticas nacionales con ciertos estándares que todos han de aceptar, bien por motivos estratégicos o bien porque éstos refuerzan la legitimidad de las decisiones. Ahora bien, fijar y aceptar estándares comunes significa introducir cambios que, de un modo u otro, terminan influyendo sobre las políticas de cada uno de los países. Es decir, crear procedimientos, normas, prácticas es también una manera de «crear Europa», a pesar de que no exista todavía un estado europeo ni un modelo político bien definido. La soberanía nacional sigue en pie, seguirá estándolo por un tiempo, aunque existen también líneas de convergencia y de ajuste mutuo entre las instituciones de la Unión. Estos procedimientos de ajuste y de negociación dibujan un panorama algo distinto, de carácter postnacional y encaminado hacia una soberanía compartida. El «solapamiento» ${ }^{23}$ de algunas políticas resulta deseable para áreas estratégicas -justicia, seguridad, moneda única, etc.- demostrando, de paso, que el sistema y el método comunitario no tienen por qué favorecer el centralismo, ya que la subsidiariedad ${ }^{24}$ sigue siendo un principio fundamental en la toma de decisiones que afectan a la Unión, a sus instituciones y a los ciudadanos.

En los últimos años, las discusiones sobre la arquitectura variable del proyecto europeo se han centrado en el tema de la ampliación y en el de las competencias de los estados miembros. Sin embargo, la gobernanza sin gobierno y las formas de «gobierno mixto» que emergen del ajuste entre políticas públicas nacionales han tenido, tal vez, mayor influencia sobre los procedimientos y las prácticas -las buenas prácticas- que sobre las instituciones nacionales y comunitarias. De hecho, los cambios y negociaciones entre países han llegado antes a áreas que son vitales para los ciudadanos, como la salud, que a los gobiernos de los distintos países. ¿Por qué razón ha sido así? ¿Cómo es posible que, hasta ahora, el destino del modelo postnacional haya influido tanto sobre decisiones

23 W. Streek subrayaba el «solapamiento» de los acuerdos y de la coordinación europea en temas específicos, «Neo-Voluntarism: A Nwe European Social Policy Regime?», Marks, G., Scharpf, F., Schmitter, Ph.: Governance in the European Union, 64-94.

24 S. Geiger se ha ocupado de las formas de gobierno no centralistas y la subsidiariedad, «Neue Formen der Governance für die Europäische Union», Europäische Governance, Tectum, Marburg, 2005, 22-50. 
con impacto sobre la calidad de vida e influya tan poco sobre la retórica política? En la Unión, las ventajas y las dificultades para la convergencia son bastante visibles en sectores como éste, debido a que el proceso de «europeanización» incide de forma directa sobre las políticas sociales de cada uno de los países, aunque tenga escaso peso en el discurso político. La complejidad, la improvisación en ciertos temas -o la falta de previsión en otros- son poco interesantes para la comunicación política estándar, sin duda. Otro tipo de análisis revela, no obstante, que la integración va en varias direcciones, con ritmos distintos y con resultados también dispares. «Europeanización» ${ }^{25}$ se refiere a esto, significa que el desarrollo de la Unión afecta a las decisiones e instituciones, a las comunitarias y a las nacionales, ya que los procesos de integración que están en marcha desencadenan o desencadenarán cambios internos en los países miembros, no sólo cambios en la arquitectura general de la Unión.

\section{PoLíticAS DE «EUROPEANIZACIÓN»}

La Europa de la salud ofrece algunas de la claves para comprender la emergencia de un estilo de gobierno que introduce cambios dentro de la estructura de los estados nacionales, además de aquellos elementos que favorecen la convergencia interestatal. Ambos niveles, lo estatal y lo supraestatal, están reflejados en las normas y en los procedimientos que regulan la práctica clínica y la investigación biomédica en el espacio de la Unión. Por esta razón, es un buen punto de partida para comprender la peculiar dinámica de la convergencia europea. Así lo indican los convenios, las resoluciones y los acuerdos europeos en estas materias, ya que intentan promover una regulación aceptada por todos los países y, al mismo tiempo, respetar las diferencias nacionales y culturales. La salud es, por tanto, un área bastante significativa, nada marginal en el proceso de «europeanización ${ }^{26}$ en su fase actual. Por ejemplo, los acuerdos sobre estos temas demuestran que ciertas formas de desnacionalización están en marcha, a pesar de las limitaciones institucionales y culturales, a pesar incluso de las diferencias entre los sistemas de salud y entre las políticas públicas europeas. Ahora bien ¿hasta qué punto es correcto afirmar que la integración de la «Europa de la salud» puede ir por delante de la de la «Europa de los ciudadanos»? Hay varias razones para pensar que las negociaciones o el «solapamiento» arrojan mejores resultados en un caso que en otro:

25 Vink, M.: «What is Europeanization? And Other Questions on a New Research Agenda», Paper YEN Research Meeting on Europeanization, November, 2002.

26 M. Steffen, W. Lamping y J. Lehto se han ocupado de la «Europa de la salud» como ejemplo de europeanización, «Introduction. The Europeanization of Health Policies», Steffen, M.: Health Governance in Europe, Routledge, London, 2005, 1.17. 
- La interdependencia, la defensa de derechos fundamentales, las necesidades de los ciudadanos, la adopción de estándares comunes o de buenas prácticas, pocas áreas como ésta ejemplifican las políticas de alcance europeo. Las muestras son muy numerosas, desde la forma de regular la donación de tejidos, los trasplantes de órganos, la investigación con sujetos humanos, las funciones que se atribuyen a los comités de ética en la mayor parte de los países, hasta los requisitos que deben cumplir aquellos proyectos de investigación que acudan a la financiación con fondos europeos. A partir de 1998, los temas bioéticos han sido objeto de atención constante por parte de varias instituciones, sobre todo del Consejo de Europa. Tampoco cabe duda de que estas cuestiones están en la agenda de todos los gobiernos, suya es la responsabilidad de organizar los sistemas de salud y velar por la protección de los consumidores. La Bioética es un ámbito multidisciplinar, complejo, tal vez por eso ha estado muy presente en la política nacional y, al mismo tiempo, en las políticas comunitarias. ${ }^{27}$ De hecho, las intervenciones normativas proceden tanto de un nivel como del otro, de manera que, a día de hoy, es una de las áreas más estructuradas en Europa. Los esfuerzos por institucionalizar la valoración ética de los avances en Medicina y Biología se han concretado también en la creación de grupos, proyectos y comités de expertos y, desde los años noventa, el Comité de Bioética de la Unión.

- Sin embargo, los acuerdos y la desnacionalización de todo lo relacionado con la salud se han producido de forma discontinua, sin un plan diseñado con anterioridad. Sin duda, los temas sanitarios favorecen la convergencia, puesto que las normas tienen alcance supranacional, también lo tienen las epidemias y los problemas de salud pública, mientras que los controles y las iniciativas proceden aún de los gobiernos nacionales. Así es la europeanización, ${ }^{28}$ se trata de un proceso de adaptación entre las instituciones nacionales que, luego, genera cambios en las políticas locales. En el caso de la Unión, los ajustes siguen una «geometría variable»-sobre todo en temas sanitarios, por la distinta configuración de los sistemas nacionales-, respetando la autonomía y, a la vez, creando reglas e instituciones compartidas. En la práctica, los países se ven obligados a modificar su legislación y sus políticas locales, con objeto de adecuarse a los nuevos estándares europeos. No

27 F. Lafond se ha referido a la Bioética como un área fundamental de europeanización, «Towards a European Bioethics Policy? », Steffen, M.: Health Governance in Europe, 152-173.

28 Olsen, J.: «The Many Faces of Europeanization», ARENA Working Papers, 01/2, $1-34$. 
habrá presiones directas para ello, si bien los acuerdos y el reconocimiento mutuo dejarán poco margen para las iniciativas a nivel local o nacional. La movilidad de los pacientes, la prestación de servicios, el reconocimiento de los títulos profesionales y la protección de los derechos de los ciudadanos en esta materia ejemplifican la incipiente coordinación de las políticas sociales dentro de la Unión.

- La salud empieza a ser un área con menos fronteras para los europeos. Además, evidencia que la integración de «geometría variable» puede ser de dos tipos, negativa y positiva. ${ }^{29}$ La primera consiste en eliminar restricciones y controles, en especial el control de fronteras. En aras de la circulación de bienes y personas, los estados han de reducir su presencia en ámbitos como la salud -también la seguridad y la protección de los consumidores-, que van más allá de lo local y lo nacional. La integración positiva muestra que lo supranacional exige más esfuerzos, que se han de crear las condiciones para la cooperación entre instituciones y gobiernos. Por tanto, la desaparición de fronteras y de reglas de alcance local tendría que ir acompañada de una nueva regulación, aceptada por todos. En este caso, el marco normativo debe tener validez general, estando más cerca de los derechos humanos que de las leyes y acuerdos de alcance nacional. En este contexto, la legitimidad para introducir los cambios oportunos solo puede estar en los sistemas abiertos, participativos, transparentes. El concepto de gobernanza responde estos criterios y, ante todo, responde a los cambios que han iniciado los países de la Unión.

- En octubre de 2007, la Comisión Europea daba a conocer el plan estratégico ${ }^{30}$ para abordar de forma conjunta los temas sanitarios. El documento reconocía que los estados son todavía responsables de las políticas de salud, la Comunidad no tiene que duplicar los esfuerzos que se realicen en este sector. Ahora bien, la cooperación entre estados resulta indispensable cuando los problemas tienen un impacto internacional y van más allá de las fronteras, situaciones que el plan tenía muy en cuenta. Por lo tanto, las políticas comunes deben ser definidas y mejoradas de forma solidaria, con la intención de hacer frente a algunos retos importantes, como el envejecimiento de la población, las nuevas amenazas para la salud -epidemias, bioterrorismo, efectos

29 Scharpf, F.: «Negative and Positive Integration in the Political Economy of European Welfare States», Marks, G., Scharpf, F., Schmitter, Ph.: Governance in the European Union, 15-39.

30 Comisión of the European Communities, White Paper. Together for Health: A Strategic Approach for the EU 2008-2013, Brussels, 23.10.2007 COM (2007) 630 final. 
negativos del cambio climático- y las dificultades que plantea el uso de las nuevas biotecnologías. El objetivo general era definir una nueva estrategia, válida hasta el año 2013, permitiendo recabar información contrastada sobre salud en todos los países de la Unión y, sobre todo, ayudando a tomar decisiones de forma consensuada, en beneficio de los ciudadanos y de su salud. No era menos importante el propósito de reducir las desigualdades en el acceso a la atención sanitaria. Por último, el plan asumía que la salud es un factor básico de desarrollo, los gastos en este sector no han de representar una carga económica sino una inversión de futuro. En el mismo año, el Parlamento Europeo ${ }^{31}$ aprobaba el segundo programa comunitario sobre salud pública, destinado a completar y a aportar financiación adicional para las políticas de los estados miembros.

Puede decirse, entonces, que los criterios de buen gobierno son mucho más que principios válidos, ya que dibujan un marco para intervenciones de alcance postnacional y, también, para definir políticas públicas más justas y más adecuadas a las necesidades de los ciudadanos. La Declaracion de Laeken ponía el acento en esto último, la necesidad de mayor sintonía entre las instituciones de la Unión y las expectativas y demandas de los europeos. El método comunitario consiste precisamente en ir en las dos direcciones, desde arriba y desde abajo, respetando la diversidad de las políticas y de los sistemas nacionales. En suma, es cierto que la gobernanza al estilo europeo no cuenta con una autoridad ni con un gobierno central, sigue incluso una dinámica fragmentaria - la geometría variable -, pero adopta un punto de vista ${ }^{32}$ bien definido a fin de avanzar hacia la Europa de los ciudadanos: con apertura, participación, responsabilidad, eficacia y coherencia.

\section{IV. «Eppur si MUOVE». Algunas CONClusiones}

La presente Carta reafirma, respetando las competencias y misiones de la comunidad y de la Unión, así como el principio de subsidiariedad, los derechos reconocidos especialmente por las tradiciones constitucionales y las obligaciones internacionales comunes de los Estados miembros. ${ }^{33}$

31 «The Programme shall complement, support and add value to the polices of the Member Status and contribute to increased solidarity and prosperity in the European Union by protecting and promoting human health and safety and improving public health», Decision No 1350/2007/EC OF THE EUROPEAN PARLIAMENT AND THE COUNCIL, of 23 October 2007, art. 2.

32 Comisión de las Comunidades Europeas, La gobernanza europea. Un libro blanco, COM (2001) 428 final.

33 Carta de Derechos Fundamentales de la Unión Europea 2007/C303/01, Preámbu- 
La Carta de Derechos del año 2007 reconoce el valor de la diversidad, también el de las libertades y derechos que son fundamentales para todos los europeos. La estructura política no está aun definida, más allá de lo que se refiere al Estado de derecho y a la democracia. El debate sobre el futuro de la Unión se ha centrado, por lo general, en el diseño general del proyecto y en los criterios de buen gobierno o gobernanza; de acuerdo con esto, el modelo postnacional y cosmopolita pone el acento en la Europa de los ciudadanos y en los sistemas democráticos. Sin embargo, la construcción del proyecto ha encontrado dificultades importantes, en los mismos países europeos y en el ámbito internacional, empezando por el hecho de que, en determinadas situaciones -la guerra de Irak, la crisis financiera internacional-, no ha existido una única voz europea, tampoco ha habido convergencia entre las políticas nacionales. Aun así, están en marcha negociaciones y procesos de ajuste que afectan o van a afectar a las políticas públicas, en cada uno de los países.

El núcleo de la integración y de la construcción europea está, primero, en el marco normativo que define las líneas básicas de una gobernanza sin gobierno. Por lo dicho, está también en las políticas sociales, que son nacionales y, cada vez más, políticas comunitarias. La «Europa de la salud» es, por tanto, un caso claro de integración a distintos niveles, ya que se realiza de manera asimétrica, fragmentaria y pragmática -se trata de resolver problemas comunes, situaciones de emergencia, epidemias, etc. -, al mismo tiempo crea normas, procedimientos, reconocimiento mutuo de libertades y de derechos. Además, el esfuerzo por llegar a acuerdos en este campo indica que la construcción europea no es sólo un proyecto intergubernamental, sino un plan para intervenir en lo próximo, en la vida cotidiana, a fin de asegurar la salud y el bienestar de los europeos. Prueba de ello es que el plan estratégico para los años 2008-2013 se ha fijado varios objetivos, como son la protección frente a las enfermedades, la mejora de la salud y la eliminación de las desigualdades que todavía existen en este ámbito. ¿Europa? Eppur si muove.

En este momento, Europa significa, quizás, más derechos, más colaboración entre gobiernos, más estándares comunes y menos -un poco menos- nación. La regulación, el énfasis en los derechos de los ciudadanos aspira a impedir abusos, como los que se cometieron en otra época -una época infame-, en nombre de la ciencia y del interés de la sociedad. En realidad, la expansión de los derechos forma parte de las lecciones que los europeos han aprendido de la Historia, a costa de sufrimientos indecibles, para no repetir errores trágicos. Las buenas prácticas de hoy son, en definitiva, el resultado de las malas prácticas del pasado, que no deben volver. La prioridad de los derechos individuales sobre otros bienes públicos explica asimismo el interés por regular al máximo todo lo que afecta

lo. 
a la salud y a la investigación, como sucede en la legislación de varios países -en España, la Ley 14/2007- sobre investigación biomédica. La preferencia por lo normativo, el interés por establecer los requisitos y los controles para que la investigación siga estándares de calidad y de moralidad, la difusión de buenas prácticas y la institucionalización de los comités de ética, todo ello forma parte del estilo europeo, centrado en la protección de los derechos individuales. Por estas razones, la Carta de Derechos se refiere en modo expreso a la Medicina y la Biología, al consentimiento informado y a la prohibición de prácticas eugenésicas. Por último, la construcción de una Europa de la salud demuestra que el cambio político puede adelantarse al cambio en las instituciones, respetando además el doble ritmo del proceso de «europeanización», entre lo nacional y lo supranacional.

\section{REFERENCIAS BIBLIOGRÁFICAS}

CARTA DE DERECHOS FUNDAMENTALES DE LA UNIÓN EUROPEA 2007/ C303/01. Convenio para la protección de los derechos humanos y la dignidad del ser humano con respecto a las aplicaciones de la Biología y la Medicina, Oviedo, 1997.

COMISIÓN DE LAS COMUNIDADES EUROPEAS, La gobernanza europea. Un libro blanco, COM (2001) 428 final.

COMISIÓN OF THE EUROPEAN COMMUNITIES, White Paper. Together for Health: A Strategic Approach for the EU 2008-2013, Brussels, 23.10.2007 COM (2007) 630 final.

Declaración de Laeken, 15-12-2001.

THE EUROPEAN PARLIAMENT Decision No 1350/2007/EC of 23 October 2007.

LEY 14/2007 de Investigación biomédica, Preámbulo.

GEIGER, S.: Europäische Governance, Tectum, Marburg, 2005.

HELD, D., MCGREW, A.: Globalization/Anti-Globalization, Polity Press, Cambridge, 2002.

MARKS, G., SCHARPF, F., SCHMITTER, Ph.: Governance in the European Union, Sage, London, 1996.

OLSEN, J.: «The Many Faces of Europeanization», ARENA Working Papers, 01/2.

STEFFEN, M.: Health Governance in Europe, Routledge, London, 2005.

TELÓ, M.: Europe: A Civilian Power? Palgrave Mac Millan, London, 2006.

WALlACE, H., WALLACE, W., POLLACK, M.: Policy-Making in the European Union, Oxford University Press, Oxford, 2005.

LÓPEZ DE LA VIEJA, M.T.: «Ética de la investigación. Las buenas prácticas», Arbor, 730, 2008, 233-245

LÓPEZ DE LA VIEJA, M.T.: «Posguerra y contracultura. Identidades en la Ética aplicada», Arbor, 722, 2006, 787-795. 
Maria Teresa López de la Vieja es Catedrática de Filosofía Moral en la Facultad de Filosofía de la Universidad de Salamanca.

\section{Publicaciones recientes:}

Principios morales y casos prácticos, Tecnos, Madrid, 2000.

Ética y Literatura, Tecnos, Madrid, 2003.

Bioética y ciudadanía, Biblioteca Nueva, Madrid, 2008.

Líneas de investigación:

Filosofía moral contemporánea, éticas aplicadas, teoría feminista.

Dirección postal:

Facultad de Filosofía, Campus Unamuno, Universidad de Salamanca, 37007 Salamanca.

Dirección electrónica: tlv@usal.es 